\title{
CARACTERIZAÇÃO DO GRÂNULO DE AMIDO DE BANANAS (Musa AAA-NANICÃo E Musa AAB-TERRA)
}

\author{
M.C.J. FREITAS ; D.de Q. TAVARES
}

\begin{abstract}
RESUMO
O amido de bananas tem sido pesquisado na área de nutrição a partir da introdução do conceito de Amido Resistente. O amido de Musa AAA-Nanicão e Musa AAB-Terra foram caracterizados quanto as suas respostas fisiológicas [12]. Em continuidade, o presente trabalho estudou características físicas e morfológicas dos grânulos de amido de ambas as espécies de banana comparando-as com amido nativo de milho comercial. O amido de bananas foi extraído segundo CHIANG, CHU \& CHU [3]. A morfologia dos grânulos foi realizada após tratamento enzimático in vitro a $37^{\circ} \mathrm{C} / 24 \mathrm{~h}$ com $\alpha$-amilase pancreática. Foram efetuados os respectivos amilogramas e difractogramas de raios-X. Os grânulos de amido da Musa Tipo AAA-Nanicão apresentaram comprimento entre 30-40 $\mu \mathrm{m}$. Em Musa AAB-Terra, os grânulos, também ovais e alongados, eram um pouco menores, 20-30 $\mu \mathrm{m}$. A corrosão enzimática in vitro iniciava-se sobre a superficie anteriormente lisa e formavam estrias superficiais e apicais. A Microscopia Eletrônica de Varredura (MEV) mostrou que a hidrólise in vitro por 24 horas foi pequena e ocorria sobretudo nas camadas amorfas dos grânulos de ambas as espécies. O padrão de corrosão demonstrou-se distinto daquele ocorrido no amido de milho. As suspensões de amido de bananas ao viscosimetro demonstraram forte capacidade de hidratação e menor capacidade de retrogradação em relação ao milho, sobretudo do amido de Musa Tipo AAA-Nanicão; o amido de Musa Tipo AAB-Terra apresentou maior estabilidade de pasta. Na análise de difração de raios-X, os grânulos de bananas apresentaram padrão tipo B e C para Musa Tipo AAA-Nanicão e Musa Tipo AAB-Terra, respectivamente. Conclui-se que os amidos de Musa AAA-Nanicão e Musa AAB-Terra são estruturalmente distintos, justificando as respostas fisiológicas distintas encontradas posteriormente pelos mesmos autores. As distinções das propriedades fisicas e bioquímicas obtidas para os grânulos, embora moderadas entre si e bastante distintas em relação ao amido de milho, não explicaram a alta resistência dos amidos das bananas à digestão enzimática. Os parâmetros obtidos nas análises permitiram, pela primeira vez avaliar as características e diferenças entre grânulos de amido de espécies distintas de banana e suas relações ao amido de milho.
\end{abstract}

Palavras-chave: amido de banana; amido resistente; hidrólise enzimática.

\section{SUMMARY}

CHARACTERIZATION OF STARCH GRANULES FROM BANANAS Musa AAA-NANICÃO AND Musa AAB-TERRA. Banana starch has been studied in the area of Nutrition ever since the concept of RS was introduced. The starches of Musa Type AAA-Nanicão and Musa Type AAB-Terra were characterized according to their physiological responses [12]. As a continuation, this study presents other physical and morphological features of starch granules from both species of banana, comparing them with native starch from commercial corn. The banana starches were extracted according to CHIANG, CHU \& CHU [3]. The morphology of the granules was examined after hydrolysis in vitro pancreatic $\alpha$-amylase. The respective X-ray amylogram and diffractogram were prepared. The length of the starch granules from Musa Type AAA-Nanicão was $30-40 \mu \mathrm{m}$. The starch granules from Musa Type AAB-Terra also presenting an oval, elongated shape, were slightly shorter between $20-30 \mu m$. The enzymatic attack in vitro initiated on the once smooth surface and the corrosion caused superficial and apical grooves. A scanning electron microscopy showed that the 24 hour hydrolysis in vitro was limited, and occurred mostly in the amorphous layers of both species. The pattern of corrosion was distinctly different in the corn starch. In the viscosimeter the banana starch suspensions demonstrated a great capacity for hydration and a smaller capacity for retrogradation as compared to corn starch, especially the starch from $M u s a$ Type AAA-Nanicão; the starch from Musa Type AAB-Terra presented a greater paste stability. In the X-ray diffraction analysis, the banana starch granules were shown to be the standard type B and C for Musa Type AAA-Nanicão and Musa Type AAB-Terra, respectively. It was concluded that the structures of the starches from Musa AAA-Nanicão and Musa AAB Terra were different, justifying the different physiological responses previously found by the same authors. The distinction between the properties, both biochemical and physical, obtained for the two types of banana starch granule, although only moderate between the two, and considerably different when compared to corn starch, did not explain the high resistance of the banana starches to enzymatic digestion. For the first time, the parameters obtained in this study allow one to evaluate the characteristics and differences between the starch granules of different kinds of banana and their relation with the corn starch.

Keywords: starch from banana; resistant starch; enzymatic hydrolysis.

\section{1 - INTRODUÇÃO}

O conceito de amidos totalmente hidrolisáveis por amilases foi modificado visto que uma fração de amidos de diversas origens resistem à digestão enzimática no in-

Recebido para publicação em 01/04/2002. Aceito para publicação em 07/03/2005(000824).

DNBE C.C.S., Instituto de Nutrição "Josué de Castro", Universidade Federal do Rio de Janeiro. End.: Av. Brigadeiro Trompowsky s/n. Cidade Universitária, CEP: 21940-590, Rio de Janeiro, RJ. E-mail: cristina@nbe.ufrj.br

DEPAN, Faculdade de Engenharia de Alimentos, Universidade Estadual de Campinas. C.P.: 6121. CEP: 13083-970, Campinas, SP. A quem a correspondência deve ser enviada. testino delgado e alcançam o intestino grosso onde serão parcialmente hidrolisados por bactérias: é a fração denominada amido resistente (AR) $[1,2,7]$.

Embora a forma e o tamanho dos grânulos de amido nativo seja distinto entre as espécies vegetais, os grânulos são organizados em zonas cristalinas alternadas por outras semi-cristalinas devido à alternância dos períodos de sintese ocorridos nos amiloplastos. A cristalinidade dos amidos varia entre 15 a 45\% [13]. Diferenças estruturais do cristal, por exemplo, das lamelas de amilopectina nos blocos cristalinos e os canais amorfos presentes devem responder em parte pelos diferentes niveis de resistência à hidrólise enzimática entre os amidos. 
Vários autores demonstraram a relação da suscetibilidade enzimática do grânulo de amido nativo com a fonte botânica e/ou enzimática $[4,13,17,20]$.

A banana verde é rica (até $84 \%$ ) em AR, portanto, um conhecimento mais detalhado das estruturas de grânulos de amido de banana verde (Musa AAA-Nanicão e Musa AAB-Terra) de espécies vegetais muito próximas, poderá fornecer subsídios para o entendimento das respectivas estruturas cristalinas. O objetivo deste trabalho é analisar a morfologia dos amidos sob microscopia eletrônica e as propriedades fisicas do amido de banana $(\mathrm{Mu}$ sa AAA-Nanicão e Musa AAB-Terra).

\section{2 - MATERIAL E MÉTODOS}

\section{1 - Extração do amido}

O amido foi extraído de Musa AAA-Nanicão e Musa AAB-Terra, pelo método de CHIANG, CHU \& CHU [3]. Amido de milho comercial foi utilizado para efeito comparativo. Os amidos de Musa AAA-Nanicão, Musa AABTerra e milho continham $0,24,0,08$ e $0,05 \%$ de proteína, 0,05, 0,03 e 0,02\% de cinza, 0,04, 0,04 e 0,03\% de lípides e 84,61 e 84,81 e $1,90 \%$ de amido resistente, respectivamente [12].

\section{2 - Análises físicas}

Foram examinadas os difractogramas de raios-X, as propriedades de pasta e e a estrutura microscópica.

\subsection{1 - Difração de raios-X}

O padrão de difração de raios-X foi determinado por difratômetro de Raio X URD 6 (Zeiss). Foi utilizada radiação CuKa a 40KV 30mA, sendo a velocidade de varredura de $0,1 / 5$ segundos, sob ângulo $2 \theta$ variando de $3-35^{\circ}$.

\subsection{2 - Propriedades de pasta}

Foram determinadas através do método descrito para viscosímetro "Rapid Visco Analyser" (RVA). Suspensões de cerca de $13 \%$ (b.s.) de amido foram aquecidas entre 35 a $95^{\circ} \mathrm{C}$ observando uma elevação a $6^{\circ} \mathrm{C} / \mathrm{min}$ e então resfriadas a $50^{\circ} \mathrm{C}$. A agitação da suspensão foi mantida a $960 \mathrm{rpm}$ por $10 \mathrm{seg}$. e depois 160 rpm durante o restante de tempo do teste. A viscosidade foi expressa em unidades RVU (Rapid Visco Unit).

\subsection{3 - Digestão enzimática (in vitro)}

Os amidos (100mg de cada) foram incubados com $\alpha$-amilase pancreática purificada (Sigma $500 \mathrm{U} / \mathrm{mg}$ de amido) em $10 \mathrm{~mL}$ de tampão tris-maleato, $\mathrm{pH} 6,9$ a $37^{\circ} \mathrm{C}$ por 24 horas, sob agitação. Centrifugados, foram lavados com $40 \mathrm{~mL}$ de etanol absoluto e deixados por 1 hora em repouso, centrifugados novamente e secos a $40^{\circ} \mathrm{C}$ por 24 horas [8].

Rapid Visco Analyser (RVA) Newport Scientific, Sidney, Australia) com o auxílio do programa "Thermocline for Windows", 1995.

\subsection{4 - Microscopia Eletrônica de Varredura (MEV)}

O detalhamento da morfologia dos grânulos foi obtido ao microscópio eletrônico de varredura (MEV) após as seguintes etapas: amostras de amido foram desidratadas à vácuo; pequena quantidade de grânulos foi aspergida sob fita metálica adesiva de dupla face e colocada sobre suportes metálicos cilindricos. Outros lotes foram desidratados em secador de ponto crítico de $\mathrm{CO}_{2}$ (Marca Balzers). Para conferir condutividade ao amido, todas as amostras foram recobertas com ouro em metalizador à vácuo (Marca Balzers). As micrografias foram obtidas no microscópio Jeol (T-300) sob aceleração de $15 \mathrm{kV}$ usando-se filme Neopan SS 135mm, 100 ASA.

\section{3 - RESULTADOS E DISCUSSÃO}

Os resultados serão apresentados para as duas espécies de bananas e para o amido de milho considerado neste trabalho como um ponto de referência de estrutura de amido de cereal.

\section{1 - Matéria-prima e extração do amido}

A polpa de Musa AAA-Nanicão era relativamente mais dura e possuía maior quantidade de sementes o que dificultou o processo convencional de separação do amido. O rendimento final de extração do amido foi 5 e $8 \%$ para Musa AAA-Nanicão e Musa AAB-Terra, respectivamente. Estas quantidades são semelhantes aos rendimentos obtidos por LII et al. [15] para amidos de bananas em estágio inicial de maturação (frutos extremamente verdes).

\section{2 - Análise física}

\subsection{1 - Difractograma de raios-X}

Os grânulos de amido Musa Tipo AAA-Nanicão apresentaram difractograma padrão B e Musa AAB-Terra padrão tipo $C$ (Figura 1). Os padrões do tipo $B$ e $C$ tendem a ser mais resistentes à amilase pancreática, conforme demonstrado nas micrografias (Figura 3). O padrão de difração da amido de milho foi tipo A (Figura 1).

Os difractogramas indicaram diferentes padrões de cristalinidade para o amido de Musa AAB-Nanicão, Musa AAA-Terra e o amido de milho comercial.

Surpreende que estas duas espécies de bananas tenham apresentado padrões diferenciados de cristalinidade visto que o teor de AR de ambas in vitro seja em torno de $84 \%$, conforme se apresentará adiante. Ė provável que a resistência de amidos com elevado teor de AR dependam de vários fatores além do tipo de associação estabelecida entre amilopectina e amilose. Esta associação entretanto é relevante até para definir o padrão de cristalinidade. De fato, segundo EERLINGEN, DECEUNINCK $\&$ DELCOUR [5], cadeias longas e compactas de amilopectina apresentam alta cristalinidade (padrão B) a qual é reforçada por numerosas associações com moléculas menores e espiraladas de amilose. Quando a amilose se 
encontra distanciada da amilopectina o grânulo tenderá a apresentar baixa cristalinidade (padrão A) e maior suscetibilidade à amilase.

\subsection{2 - Propriedade de pasta}

A Figura 2 demonstra as propriedades de pasta dos amidos: A Musa AAA-Nanicão apresentou menor temperatura de pasta $\left(71,06^{\circ} \mathrm{C}\right)$ elevando-se para Musa AABTerra $\left(74,50^{\circ} \mathrm{C}\right)$ e amido de milho $\left(78,45^{\circ} \mathrm{C}\right)$. A viscosidade inicial de pasta refletiria o grau de resistência dos grânulos, resistência esta explicada pelo tamanho das moléculas de amilose [9, 13, 14]. O amido de milho apresentou propriedades de pasta semelhantes aos da literatura $[10,19]$.

As suspensões de amido de bananas quando submetidas a aquecimento progressivo apresentaram aumento da viscosidade, e o milho, por sua vez, atingiu apenas $60 \%$ da viscosidade das bananas (Figura 2).
Os dois amilogramas das bananas verdes demonstraram viscosidade máxima elevada, indicando resistência análoga dos grânulos para ambas. Após a viscosidade máxima, a fragmentação foi abrupta para as bananas. O gel de amido de milho demonstrou menor resistência à fragmentação e por sua vez a retrogradação do gel de milho superou a retrogradação das bananas.

De fato, vários trabalhos [13,14,18,19], demonstram que resultados semelhantes aos obtidos com os amilogramas de bananas são devidos à coesão e estabilidade do grânulo durante o aquecimento.

Os resultados obtidos no estudo das propriedades de pasta com o viscosimetro (RVA) são coerentes com os difractogramas e indicam maior flexibilidade e menor coesão do amido de milho (padrão A) em relação ao amido de banana (padrão B e C). Dessa forma, o amido de milho comercial é mais facilmente danificado, frágil portanto, os
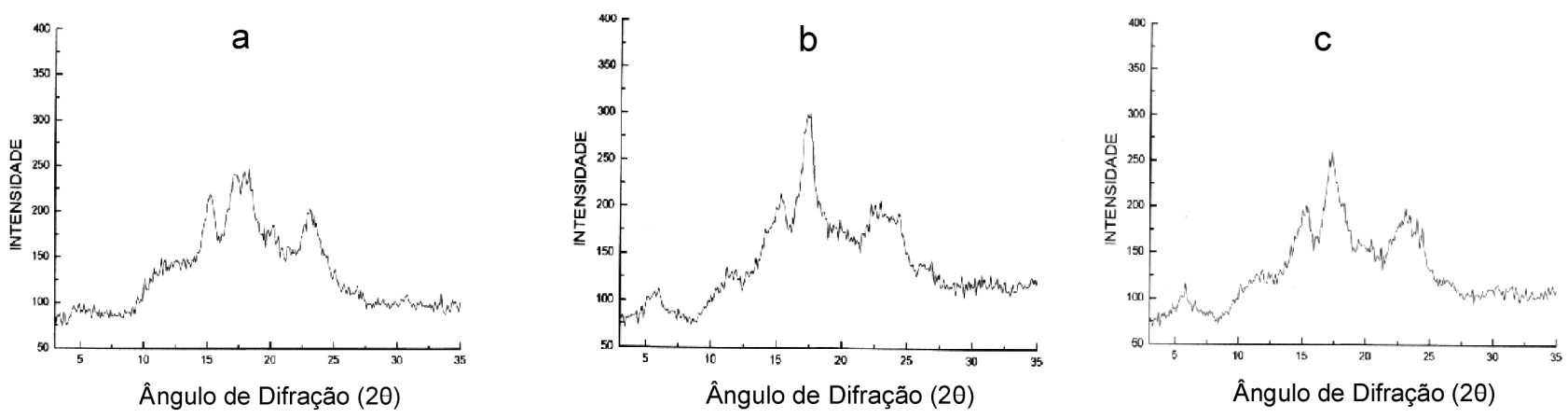

FIGURA 1 - Difractograma de raio-X: a: amido de Milho: padrão A; b: amido de Musa AAA-Nanicão: padrão B; c: amido de Musa AABTerra: padrão C
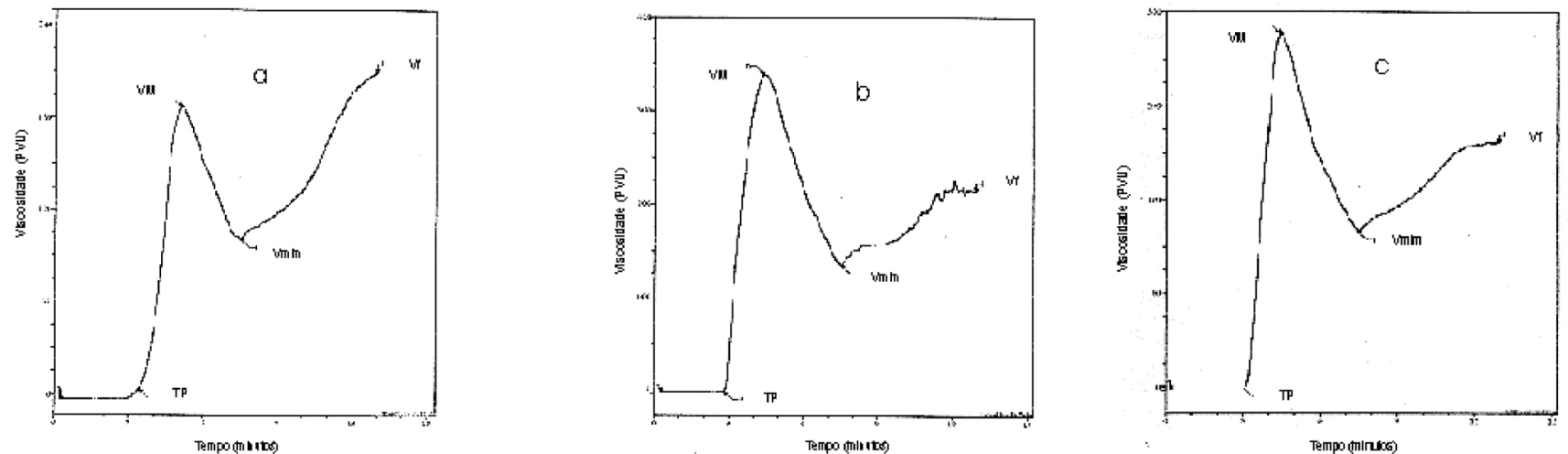

Onde:

$\mathrm{VM}=$ Viscosidade máxima em unidades RVU

Vmin $=$ Viscosidade minima a $95^{\circ} \mathrm{C} \mathrm{em} \mathrm{RVU}$

$\mathrm{VF}=$ Viscosidade a temperatura de $50^{\circ} \mathrm{C}$ em RVU

$\mathrm{TP}=$ Temperatura de pasta ${ }^{\circ} \mathrm{C}$

RVU = Unidade do RVA

FIGURA 2 - Propriedades de pasta dos amidos obtidas no viscosimetro RVA: a: amido de Milho; b: amido de Musa AAA-Nanicão; c: amido de Musa AAB-Terra 
grânulos são atacados mais facilmente por agentes físicos, químicos e biológicos.

\subsection{3 - Digestibilidade in vitro dos amidos}

A ação das amilases sobre o amido de diferentes fontes continua sendo uma questão de investigação. A elevada hidrólise do amido de milho pela $\alpha$-amilase pancreática suína está demonstrada na Figura 3 e contrastou-se com a reduzida amilólise dos grânulos de amido das duas bananas, as quais demonstraram $84 \%$ de resistência. No milho, a corrosão pela $\alpha$-amilase foi radial e por vezes completa sendo análoga a vários outros trabalhos $[9,12,13,16]$. A corrosão dos grânulos de amido de bananas foi sobretudo superficial e eventualmente apical (Figuras 3 e 4) demonstrando a excepcional resistência dos amidos das duas espécies.

A diferença na suscetibilidade amilolítica entre o amido de milho e o de bananas sugere diferenças do padrão de cristalinidade das regiões cristalinas. $\mathrm{O}$ amido de milho deve apresentar moléculas mais frouxamente ligadas,superficie áspera e formas poliédricas, elementos que favoreceriam o ataque enzimático (Figura 3). A respeito desses elementos HOOVER \& SOSULSKY [14] postularam que grânulos mais suscetiveis à amilase são porosos, tem superficies ásperas e mesmo estruturas semelhantes a esponjas apresentando canais que facilitariam
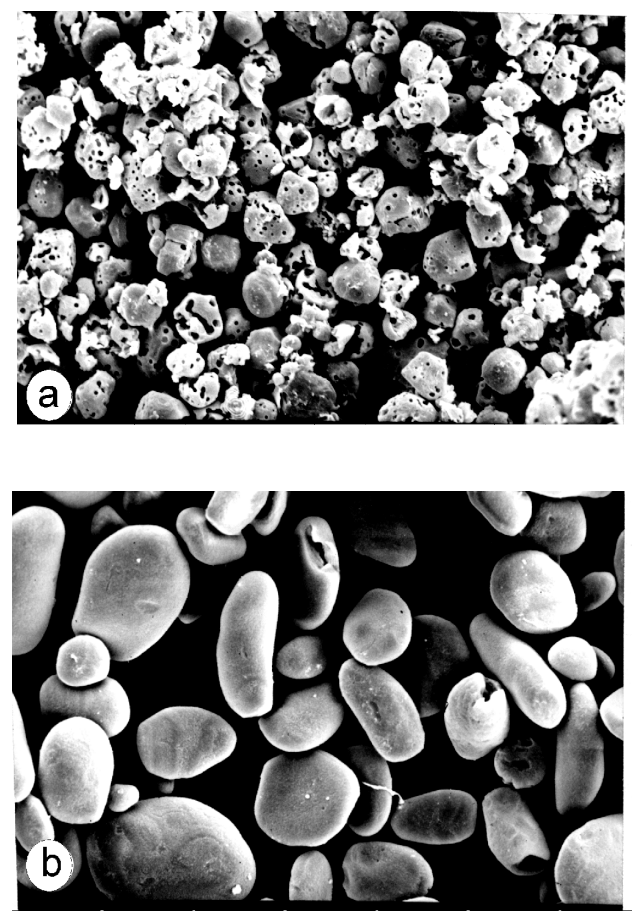

FIGURA 3 - Em A micrografias ao MEV do amido de milho tratado com $\alpha$-amilase pancreática $(800 x)$; grânulo suscetivel a ação enzimática apresentando bastante corroído com poros numerosos e profundos. Em B micrografia do amido de banana Musa AAB-Terra tratado com $\alpha$-amilase pancreática (1.000x); os grânulos demonstraram ser resistentes à enzima e a corrosão foi sobretudo superficial e apical a penetração da amilase.

GALLANT, BOUCHET \& BALDWIN [13] relataram que a resistência do amido de batata poderia estar relacionada à condensação molecular em regiões organizadas como "blocos" sobretudo de amilopectina. Prosseguindo, os autores, responsabilizam também a localização das áreas amorfas e suas relações nas cadeias e blocos de amilopectina as quais, definirão as seguintes propriedades do amido: coesão de pasta, elasticidade e variações no volume granular quando submetidos à hidratação sob aquecimento.

\subsection{4 - Microscopia Eletrônica de Varredura (MEV)}

Entre os grânulos das duas variedades de banana predominaram formas lisas, ovais, alongadas e tamanhos diferenciados (Figuras $3 b$ e 4b). Formas similares foram reportadas por LII et al. [15] e EGGLESTON, SWENNEN \& AKONI [6]. As micrografias em MEV não mostraram diferenciação superficial entre os amidos naturais de ambas as bananas, porém, observa-se menor grau de hidrólise nos amido de Musa AAA-Nanicão em relação a Musa AAB-Terra (Figuras 3 e 4).

Os grânulos de amido de milho natural apresentaram formas poliédricas e polimorfismo (Figura 4a). O ami-
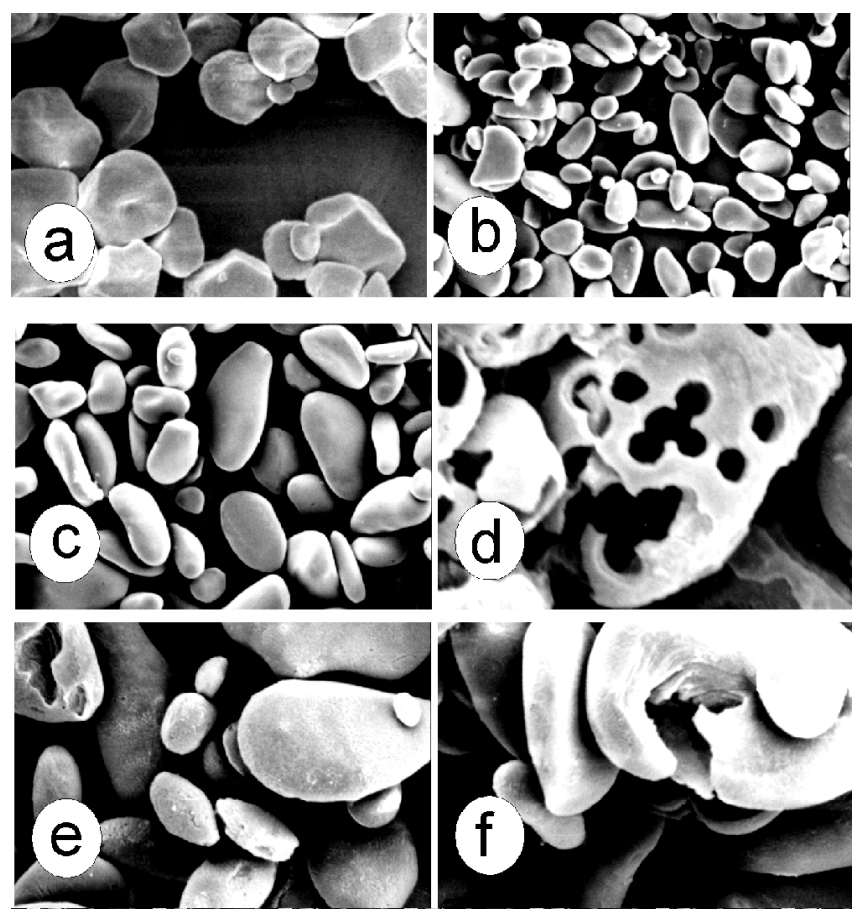

FIGURA 4 - Micrografia em MEV do amido nativo de milho e Musa AAA-Nanicão e Musa AAB-Terra e hidrolisados pela $\alpha$ amilase pancreática: a: amido de milho; b: amido Musa AAANanicão (330x); c: amido Musa AAB-Terra (430x); as micrografias seguintes são de amidos tratados com $\alpha$-amilase in vitro; d: amido de milho (3.300x); e: amido de Musa AAANanicão (1.000x); f: amido de Musa AAB-Terra (1.100x) 
do de milho tratado com $\alpha$ - amilase pancreática tem imagens ao MEV características para grânulos de alta suscetibilidade à ação enzimática, corrosão acentuada e inúmeros poros atravessando o grânulo determinando fraturas totais, como foi já descrito na literatura [10,21]. Esta característica está ligada ao maior diâmetro como demonstrado por FRANCO, CIACCO \& TAVARES [11]. Os grânulos de amido de Musa AAB-Terra apresentaram paralelamente este padrão (Figura 4d), enquanto que os grânulos de amido de Musa AAA-Nanicão observaram-se endocorrosões côncavas nos grânulos menores e exocorrosões, sobretudo apical nos grânulos maiores (Figura 3e).

Superficies do grânulo nativo de diferentes fontes botânicas como textura áspera ou alterações provocadas durante o processo de extração e purificação (fissuras), bem como a forma também pode facilitar o ataque enzimático; as formas esféricas dos grânulos resistem mais ao ataque em relação aos grânulos de forma poliédrica [20].

\section{4 - CONCLUSÕES}

Foram demonstradas diferenças físicas e morfológicas entre os amidos de Musa Tipo AAA-Nanicão e Musa Tipo AAB-Terra. A extração do amido de Musa Tipo AABTerra foi mais eficiente e facilitada pelas características estruturais da polpa. Verificou-se elevada resistência à $\alpha$-amilase pancreática nos amidos das bananas estudadas. As propriedades de pasta indicaram maior resistência à ação mecânica dos amidos de bananas padrão B e C em relação ao amido de milho padrão de cristalinidade tipo A.

Demonstrou-se que os amidos de duas espécies próximas de bananas (verdes) apresentaram baixa suscetibilidade à $\alpha$-amilase in vitro (84\%), difractogramas distintos (B e C), propriedade de pasta e retrogradação semelhantes. As propriedades fisicas demonstraram dois amidos altamente resistentes e distintos em relação ao amido de milho, demonstrando que a diferenciação de espécies abriga também diferenciações de estruturas cristalinas.

\section{5 - REFERÊNCIAS BIBLIOGRÁFICAS}

[1] ASP, N-G.; TOVAR, J.; BAIROLIYA. Determination of resistant starch in vitro with different methods and in vivo with a rat model. European Journal of Clinical Nutrition, v. 46, n.2, p.117-19, 1992.

[2] CHAMP, M.; FAISANT, N. Resistant starch: analytical and physiological aspects. Boletim da Sociedade Brasileira de Ciênc. Tecnol. Aliment. São Paulo, v.30, n.1, p.37-43, jan/jun, 1996.

[3] CHIANG, B.H.; CHU, W.C.; CHU, C.L. A pilot scale study for banana starch production. Starch/Stärke. Weinheim, v.39, n.1, p.5-8, 1987.

[4] COLONNA, P.; LELOUP,V.; BULEON, A. Limiting factors of starch hydrolysis. European Journal of Clinical Nutrition. London, v.46, n.2, p.17-32, 1992.
[5] EERLINGEN, R.C.; DECEUNINCK, M.; DELCOUR, J.A. Enzyme-resistant starch II. Influence of amylose chain length on resistant starch formation. Cereal Chemistry, St. Paul, v.70, n.3, p.345-50, 1993.

[6] EGgleston, G.; SWENNEN, R.; AKONI, S. Physicochemical studies on starches isolated from plantain cultivars, Plantain hybrids and cooking bananas. Starch/Stärke. Weinheim, v.44, n.4, p.S1218, 1992.

[7] ENGLYST, H.N.; KINGMAN, S.M.; CUMMINGS, J.H. Classification and measurement of nutritionally important starch fractions. European Journal of Clinical Nutrition. London, v.46, n.2, p.33-50, 1992.

[8] FAISANT, N.; PLANCHOT, V.; KOZLOWSKI, F.; PACOURET, M.P.; COLONNA, P.; CHAMP, M. Resistant starch determination adapted to products containing high level of resistant starch. Sciences des Aliments, Paris, v. 15, n.1, p.83-9, 1995.

[9] FRANCO, C.M.L.; CIACCO, C.F. Factors that affect the enzymatic degradation of natural starch granules: Effect of the size of the granules. Starch/Stärke. Weinheim, v.44, n.11, p. 422- 6, 1992.

[10] FRANCO, C.M.L.; CIACCO, C.F.; TAVARES, D.Q. Studies on the susceptibility of granular cassava and corn starches to enzymatic attack. Part 2. Study of the granular structure. Starch/Stärke.Weinheim, v.40, p.29-32, 1988.

[11] FRANCO, C.M.L.; CIACCO,C.F; TAVARES, D.Q. The structure of waxy corn starch: Effect of granule size. Starch/Stärke. Weinheim, v.50, n.5, p.193-8S, 1998.

[12] FREITAS, M.C.J.; TAVARES, D.Q. Caracterização dos grânulos de amido de bananas (Musa AAA-Nanicão e Musa AAB-Terra). XIV Congresso Brasileiro de Ciência e Tecnologia de Alimentos. Rio de Janeiro, v.1, p.56972,1998 .

[16] Gallant, D.J.; BOUCHET, B.; BALDWIN, P.M. Microscopy of starch: evidence of a new level of granule organization. Carbohydrate Polymers. Barking,v.32, p.177-91, 1997.

[17] HOOVER, R.; SOSULKY, F.W. Composition, structure, funcionality and chemical modification of legumes starches: a review. Canadian Journal of Physiology and Pharmacology. Ottawa, v.69, p.79-92, 1991.

[18] LII, CHENG-YI; CHANG, SHUH-MING; YOUNG, YA-LAN. Investigation of the physical and chemical properties of banana starches. Journal of Food Science. Chicago, v.47, n. 8, p.1493-97, 1982.

[19] NORDIN, P.; MOSER, J.; RAO, G.; GIRIAND, N.; LIANG, T. Labeling of starch granules by bombardment with tritium atoms. Starch/Stärke. Weinheim,.22, p.356, 1970.

[20] PLANCHOT, V.; COLONNA, P.; GALLANT, D.J.; BOUCHET, B. Extensive degradation of native starch granules by alpha-amylase from Aspergillus fumigatus. Journal of Cereal Science, London, v.21, p.163-171, 1995.

[21] SHANTHA, H.S.; SIDDAPPA, G.S. Physicochemical nature of banana Pseuds term starch. Journal Food Science. Chicago, v.35, p.72-4, 1970. 
[22] TEIXEIRA, M.A.V.; CIACCO, C.F.; TAVARES, D.Q.; BONEZZI, A.N. Ocorrência e caracterização do amido resistente em amidos de milho e de banana. Ciênc. Tecnol. . Aliment., v. 18, n.2, p.246-53, 1998.

[23] VAletudie, J-C.; COlONNA, P.; BOUCHET, B.; GALLANT, D.J. Hydrolysis of tropical tuber starches by bacterial and pancreatic $\alpha$-amylases. Starch/Stärke. Weinheim, v.45, n.8, p.270-6, 1993.
[24] YAMANDA, T.; HISAMATSU, M.; TERANISHI, K.; KATSURO, K.; HASEGAWA, N.; HAYASHI, M. Components of the porous maize starch granule prepared by amylase treatment. Starch / Stärke. Weinheim, v.46, n.9, p.358-61, 1995. 